Bull. Mater. Sci., Vol. 38, No. 2, April 2015, pp. 499-509. (c) Indian Academy of Sciences.

\title{
Experimental and quantum chemical studies on corrosion inhibition performance of fluconazole in hydrochloric acid solution
}

\author{
P MALEKMOHAMMADI NOURI and M M ATTAR* \\ Department of Polymer Engineering and Color Technology, Amirkabir University of Technology, Tehran 4413-15875, Iran
}

MS received 30 July 2014; revised 14 October 2014

\begin{abstract}
The corrosion inhibition effect of fluconazole (FLU) was investigated on steel in 1 M hydrochloric acid solution. Weight loss measurements and atomic force microscope analysis were utilized to investigate the corrosion inhibition properties and film formation behaviour of FLU. Quantum chemical approach was also used to calculate some electronic properties of the molecule in neutral and protonated form in order to find any correlation between the inhibition effect and molecular structure of FLU molecule. The results showed that FLU can act as a good corrosion inhibitor for steel in hydrochloric acid solution at different temperatures and it can inhibit steel corrosion up to $95 \%$. The adsorption followed the Langmuir isotherm and the thermodynamic parameters were also determined and discussed. Quantum chemical studies showed that in adsorption process of FLU molecules, nitrogen and oxygen atoms and benzene ring act as active centres.
\end{abstract}

Keywords. Fluconazole; corrosion inhibition; AFM; chemisorption; quantum chemical studies.

\section{Introduction}

Acid solutions are commonly used for removal of undesirable scale and rust in the metal working, cleaning of boilers and heat exchangers. ${ }^{1}$ As acidic medium, the use of hydrochloric acid in pickling of metals, acidization of oil wells and in cleaning of scales is more economical, efficient and troublefree, compared to other mineral acids. Due to general aggression of acid solutions, inhibitors are commonly used to reduce the corrosive attack on metallic materials. ${ }^{2}$ The corrosion protection using inhibitors is mainly based on the modification of the metal surface through the adsorption of inhibitor molecules and the subsequent formation of a protective blocking layer. ${ }^{3,4}$

The efficiency of an organic compound as a successful inhibitor mainly depends on its ability to adsorb on the metal surface. Most organic inhibitors adsorb on the metal surface by displacing water molecules on the surface and forming a compact barrier. Heteroatoms, such as sulphur, phosphorus, nitrogen and oxygen, together with aromatic rings in inhibitor structure can act as adsorption centres that could adsorb on the metal surface and form barrier film. The planarity and the lone electron pairs in the heteroatom's are also important features that determine the adsorption behaviour of molecules on the metallic surface. ${ }^{5-9}$

Unfortunately most of the organic inhibitors are toxic and environmentally unfriendly. Thus, in recent years the development of the novel corrosion inhibitors of natural source and non-toxic type has been considered to be more important and desirable. Due to non-toxic characteristics and

\footnotetext{
*Author for correspondence (attar@aut.ac.ir)
}

negligible negative impacts on the aquatic environment, drugs (chemical medicines) seem to be ideal candidates to replace traditional toxic corrosion inhibitors. ${ }^{10-12}$

Quantum-chemistry calculations have been widely used to study the reaction mechanisms and to interpret the experimental results as well as to solve chemical ambiguities. Theoretical investigations based on quantum chemical calculations have been proposed as a powerful tool for predicting a number of molecular parameters directly related to the corrosion inhibiting property of any chemical compound. ${ }^{13,14}$

According to literature, triazole derivatives have shown good corrosion inhibition efficiency in acidic medium. ${ }^{14-16}$ Obot et $a l^{10}$ showed that fluconazole (FLU) (figure 1), as a triazole derivative, is an effective corrosion inhibitor for aluminium in acidic medium. ${ }^{17,18}$ This substance is a pharmaceutical drug, which can be classified as an antifungal drug.

The present work is aimed to determine the corrosion inhibition effectiveness and adsorption behaviour of FLU for steel in hydrochloric acid solution by gravimetric method and atomic force microscope (AFM) techniques at different temperatures. Density functional theory (DFT) was also used to calculate electronic properties relevant to the inhibiting action in order to explain inhibition efficiency.

\section{Experimental}

\subsection{Materials}

FLU was obtained from Parsdarou Pharmaceutical Co. Steel panels were prepared from FooladMobarake Co. The elemental composition of the steel is presented in table 1 . 


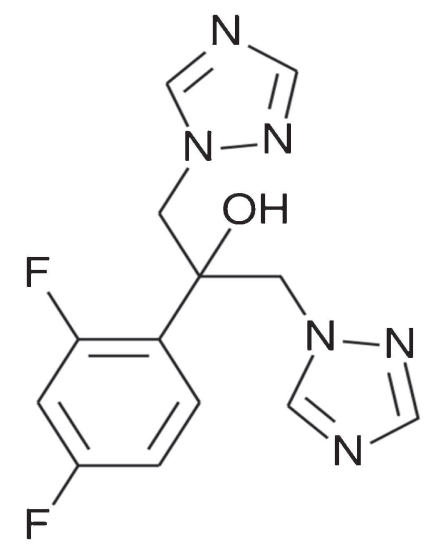

Figure 1. The chemical structure of fluconazole.

Table 1. Composition of steel panels.

\begin{tabular}{lccccccc}
\hline Elements & $\mathrm{Fe}$ & $\mathrm{C}$ & $\mathrm{Si}$ & $\mathrm{Mn}$ & $\mathrm{P}$ & $\mathrm{S}$ & $\mathrm{Al}$ \\
\hline $\mathrm{Wt} \%$ & 99.01 & 0.19 & 0.34 & 0.32 & 0.05 & 0.05 & 0.04 \\
\hline
\end{tabular}

\subsection{Gravimetric measurements}

The gravimetric method (weight loss) is probably the most widely used method of inhibition assessment. The simplicity and reliability of the measurement offered by this method means that the technique forms the baseline method of measurement in many corrosion monitoring programs. ${ }^{19}$ Weight loss measurements were conducted in the test solutions for $24 \mathrm{~h}$ at different temperatures (296-316 K). In each experiment, the pre-cleaned and weighed specimens suspended with the aid of plastic rod in a beaker containing $160 \mathrm{ml} 1 \mathrm{M}$ $\mathrm{HCl}$ solution. All tests were carried out in aerated solutions and were run in triplicate to guarantee the reliability of the results. The mean value of the weight loss was reported. To determine weight loss, the specimens were retrieved from test solutions, scrubbed with bristle brush under running water in order to remove the corrosion product, dried with hot air stream and re-weighed accurately. The weight loss was taken as the difference between the weight at a given time and the initial weight of the test coupon, determined using digital balance with sensitivity of $\pm 1 \mathrm{mg}$.

Specimens used in the weight loss experiment were mechanically cut into $5.0 \times 4.0 \times 0.15 \mathrm{~cm}$ dimensions. Specimens were mechanically ground with 320 and 600 grades of emery paper, then degreased with acetone and dried. Distilled water was used for preparing test solutions for weight loss measurements. $\mathrm{HCl}(1 \mathrm{M})$ solutions containing different concentrations of FLU, 5, 10, 25, 50, 100 and 200 p.p.m., were prepared.

\subsection{AFM analysis}

Surface morphologies of the steel panels exposed to the acidic medium were studied by Ambios Technologies USPM instrument in tapping mode.

\subsection{Quantum chemical calculation}

The quantum chemical calculation was performed using Spartan' 08V1.2.0 program. A restricted Hartree-Fock SCF calculation performed using Pulay DIIS with Geometric Direct Mini mization Geometry optimization was carried out by DFT level with the B3LYP functional at the basis set 6-31G* and water as solvent. This approach (B3LYP functional at the basis set $6-31 \mathrm{G}^{*}$ ) has shown to be adequate for predicting molecular properties and has yielded favourable geometries for a wide variety of systems. ${ }^{20,21}$ Another reason for choosing this approach was that according to Spartan program manual Hartree-Fock and MP2 models with 6-31G* and larger basis sets and density functional models, all generally pro vide good descriptions of conformational energy differences in organic compounds. The manual also state that MP2 and B3LYP density functional models are the most reliable models.

\section{Result and discussion}

\subsection{Influence of inhibitor concentration on the corrosion rate}

Figure 2 shows the variation of inhibition efficiency of 200 p.p.m. of FLU in $1 \mathrm{M} \mathrm{HCl}$ as a function of immersion time. It is evident from the figure that inhibitor efficiency increases with increasing of immersion time and it stabilizes at the value of $94 \%$ after $24 \mathrm{~h}$. The increase of inhibitor efficiency with time may be attributed to the formation of a barrier film, which prevents the attack of the metal surface by acid.

The values of corrosion rates $(v)$, inhibition efficiencies (IE\%) and the degrees of surface coverage $(\theta)$ were obtained from weight loss measurements for different concentrations of $\mathrm{FLU}$ in $1 \mathrm{M} \mathrm{HCl}$ after $24 \mathrm{~h}$ immersion at different temperatures (table 2).

The corrosion rate $(v)$ was calculated from the following equation: ${ }^{22}$

$$
v=3.45 \times 10^{6} \times\left(m_{1}-m_{2}\right) / A T D,
$$

where $m_{1}$ and $m_{2}$ are the masses of the steel coupon before and after immersion (mg), respectively, $A$ the total area of the

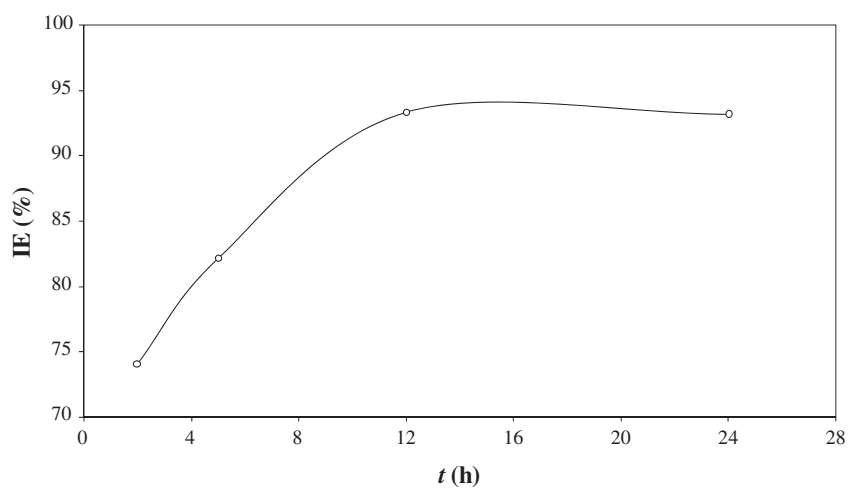

Figure 2. Variation of the inhibition efficiency with immersion time of 200 p.p.m. FLU in $1 \mathrm{M} \mathrm{HCl}$ at $296 \mathrm{~K}$. 
steel coupon $\left(\mathrm{cm}^{2}\right), T$ the corrosion time (h), $D$ the density of the steel specimen $\left(\mathrm{g} \mathrm{cm}^{-3}\right)$ and $v$ the corrosion rate (mpy). The percentage of inhibition efficiency (\% IE) was calculated using the following equation: ${ }^{23}$

$$
\% \mathrm{IE}=\left(v_{0}-v\right) / \nu_{0} \times 100,
$$

where $v_{0}$ and $v$ are the corrosion rates of the steel coupon in $\mathrm{HCl}$ solution in the absence and presence of FLU, respectively.

The results showed that FLU actually inhibited the corrosion of steel in $1 \mathrm{M} \mathrm{HCl}$ solution. The corrosion rate was found to be dependent on the concentration of the inhibitor. From table 2, it is obvious that increasing the concentration of FLU at different temperatures increased the inhibition efficiency (IE\%) up to relatively $95 \%$.

The inhibitory action of an organic inhibitor like FLU against steel corrosion can be attributed to the adsorption of inhibitor molecules on the steel surface. The degree of surface coverage of inhibitor on steel surface increased with the increase of inhibitor concentrations, which means that steel surface was separated from the corrosive medium by the inhibitor adsorption film. In fact the film formed on surface could limit the dissolution of the steel by blocking its corrosion sites. ${ }^{18,24}$

\subsection{Adsorption isotherm and thermodynamic parameters}

The metal surface in aqueous solution is always covered with adsorbed water dipoles. Therefore, the adsorption of an

Table 2. Corrosion rates, inhibition efficiencies (IE\%) and the degrees of surface for FLU in $1 \mathrm{M} \mathrm{HCl}$ after $24 \mathrm{~h}$ immersion at 296-326 K.

\begin{tabular}{|c|c|c|c|c|}
\hline $\begin{array}{l}\text { Temperature } \\
(\mathrm{K})\end{array}$ & $\begin{array}{l}\text { Concentration } \\
\text { (p.p.m.) }\end{array}$ & $\begin{array}{l}\text { Corrosion rate } \\
\text { (mpy) }\end{array}$ & $\mathrm{IE} \%$ & $\theta$ \\
\hline \multirow[t]{7}{*}{296} & 0 & 231.52 & - & - \\
\hline & 5 & 48.38 & 79.10 & 0.7910 \\
\hline & 10 & 32.25 & 86.07 & 0.8607 \\
\hline & 25 & 25.59 & 88.95 & 0.8895 \\
\hline & 50 & 17.52 & 92.43 & 0.9243 \\
\hline & 100 & 14.42 & 93.77 & 0.9377 \\
\hline & 200 & 15.82 & 93.17 & 0.9317 \\
\hline \multirow[t]{7}{*}{306} & 0 & 507.54 & - & - \\
\hline & 5 & 174.92 & 65.54 & 0.6554 \\
\hline & 10 & 123.75 & 75.62 & 0.7562 \\
\hline & 25 & 54.20 & 89.32 & 0.8932 \\
\hline & 50 & 44.43 & 91.25 & 0.9125 \\
\hline & 100 & 35.36 & 93.03 & 0.9303 \\
\hline & 200 & 30.94 & 93.90 & 0.9390 \\
\hline \multirow[t]{7}{*}{316} & 0 & 1189 & - & - \\
\hline & 5 & 436.13 & 63.32 & 0.6332 \\
\hline & 10 & 274.71 & 76.90 & 0.7690 \\
\hline & 25 & 161.43 & 86.42 & 0.8642 \\
\hline & 50 & 113.51 & 90.45 & 0.9045 \\
\hline & 100 & 77.69 & 93.47 & 0.9347 \\
\hline & 200 & 57.69 & 95.15 & 0.9515 \\
\hline
\end{tabular}

organic molecule on the surface of steel electrode is considered as a substitution of corrosion inhibitor $(I)$ with water molecules adsorbed on the steel surface: ${ }^{25}$

$$
\mathrm{M}\left(n \mathrm{H}_{2} \mathrm{O}\right)_{(\mathrm{ads})}+\mathrm{I}<=>\mathrm{MI}_{\mathrm{ads}}+n \mathrm{H}_{2} \mathrm{O}_{(\mathrm{sol})},
$$

where $I$ represents the corrosion inhibitor molecules, which displace $n$ water molecules initially adsorbed on the metal surface (M).

It is well known that the degree of surface coverage of corrosion inhibitor on metal surface $(\theta)$ is the most useful parameter for studying the adsorption behaviour of the inhibitors, which can be calculated as $\theta=\mathrm{IE} / 100$. Basic information regarding the interaction between the inhibitors and the steel surface can be provided by the adsorption isotherm. ${ }^{26}$ The most frequently used adsorption isotherms are Langmuir, Temkin, Frumkin and Freundluich. ${ }^{25}$ Attempts were made to fit $\theta$-values to these isotherms. The best fit was obtained with Langmuir isotherm according to the following equation: ${ }^{25}$

$$
\theta /(1-\theta)=K_{\mathrm{ads}} C
$$

Rearranging equation 4 gives:

$$
C / \theta=1 / K_{\mathrm{ads}}+C,
$$

where $K_{\text {ads }}$ is the equilibrium constant of adsorption reaction. The Langmuir isotherm is based on the assumption that each site of metal surface holds one adsorbed species; therefore, one adsorbed $\mathrm{H}_{2} \mathrm{O}$ molecule is replaced by one organic molecule on the steel surface. This kind of isotherm assumes that the adsorption of organic molecules occurs as a monolayer over the metallic surface without any interactions with other molecules adsorbed. ${ }^{27,28}$

$C / \theta$ against $C$ was plotted, which gave a straight line close to unit slope (figure 3 ). This indicates that the adsorption of FLU on the steel surface in $1 \mathrm{M} \mathrm{HCl}$ solution follows the Langmuir adsorption isotherm. The relationship between $C / \theta$ and $C$ at different temperatures can be expressed by equations (6-8):

$$
\begin{aligned}
& \mathrm{C} / \theta=1.066 C+0.000003\left(R^{2}=1, T=296 \mathrm{~K}\right) \\
& \mathrm{C} / \theta=1.052 C+0.000008\left(R^{2}=1, T=306 \mathrm{~K}\right) \\
& \mathrm{C} / \theta=1.037 C+0.00001\left(R^{2}=1, T=316 \mathrm{~K}\right),
\end{aligned}
$$

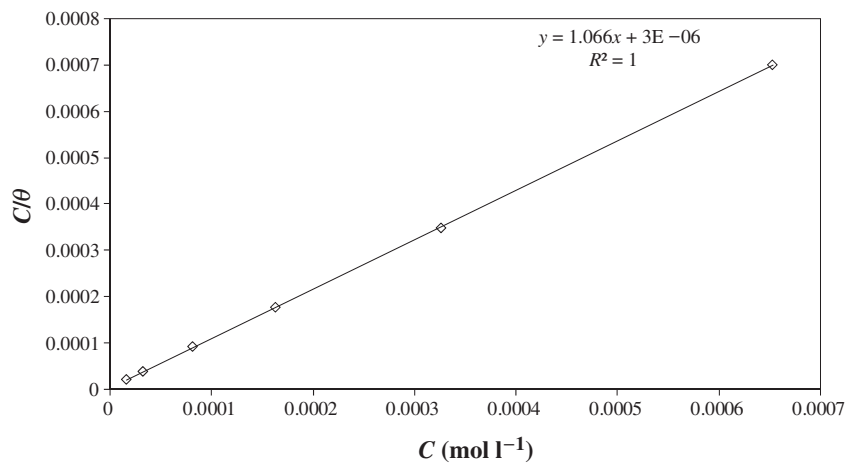

Figure 3. Langmuir adsorption plot as $C / \theta$ against $C$ for FLU at $296 \mathrm{~K}$. 
where $C$ is the concentration of FLU in $\mathrm{mol}^{-1}$. By making comparison between equations (5) and (6-8), $K_{\text {ads }}$ can be calculated. The constant $K_{\text {ads }}$ is related to the standard-free energy of adsorption $\Delta G_{\text {ads }}^{\circ}$ by following equation:

$$
K_{\text {ads }}=\frac{1}{55.5} \exp \left(\frac{\Delta G_{\text {ads }}^{\circ}}{\mathrm{RT}}\right),
$$

where $R$ is universal gas constant, $8.3144 \mathrm{~J} \mathrm{~K}^{-1} \mathrm{~mol}^{-1}, T$ is the temperature in $\mathrm{K}$. The value of 55.5 is the concentration of water in solution, expressed in moles per litre. The values of $K_{\text {ads }}$ and $\Delta G_{\text {ads }}^{\circ}$ were calculated (table 3 ).

The negative values of $\Delta G_{\text {ads }}^{\circ}$ ensure the spontaneity of the adsorption process and stability of the adsorbed layer on the steel surface. Two main types of interaction can describe the adsorption of an organic inhibitor on the metal surface: physisorption and chemisorption. Generally, values of $-\Delta G_{\text {ads }}^{\circ}$ up to $20 \mathrm{~kJ} \mathrm{~mol}^{-1}$ are consistent with electrostatic interactions between the charged molecules and charged metal (physisorption), while those around $40 \mathrm{~kJ}$ $\mathrm{mol}^{-1}$ or higher are associated with chemisorption as a result of sharing or transfer of electrons from organic molecules to the metal surface to form a coordinate type of bond. ${ }^{29,30}$ The obtained values of $-\Delta G_{\text {ads }}^{\circ}$ were above $40 \mathrm{~kJ} \mathrm{~mol}^{-1}$. Therefore, FLU molecules adsorbed on the steel surface predominately by a chemisorption method.

Langmuir equation can be written in another form: ${ }^{31}$

$$
\theta /(1-\theta)=A C \exp \left(-\Delta H_{\text {ads }}^{\circ} / R T\right),
$$

where $T$ is the temperature, $A$ is an independent constant, $C$ is the inhibitor concentration, $R$ is the gas constant, $\Delta H_{\text {ads }}^{\circ}$ is the standard heat of adsorption and $\theta$ the surface coverage by the inhibitor molecules. In order to calculate $\Delta H_{\text {ads }}^{\circ}$, the function of $\ln [\theta /(1-\theta)] v s .1 / T$ at the optimum concentration of inhibitor (200 p.p.m.) was plotted (figure 4). The slope of the linear part of the curve is equal to $\Delta H_{\text {ads }}^{\circ} / R$, from which the average heat of adsorption $\left(\Delta H_{\text {ads }}^{\circ}\right)$ can be calculated as $14.08 \mathrm{~kJ} \mathrm{~mol}^{-1}$.

The positive value of $\Delta H_{\text {ads }}^{\circ}$ shows that the adsorption of inhibitor is an endothermic process, which indicates that IE values increase with increasing the temperature. Generally, an endothermic process is attributable unequivocally to chemisorption process, while exothermic process signifies either physi- or chemisorption. ${ }^{32}$ In our work, $\Delta H_{\text {ads }}^{\circ}$ was $14.08 \mathrm{~kJ} \mathrm{~mol}^{-1}$, which suggests that FLU is adsorbed on the steel surface predominately by chemisorption method. This confirms the result obtained from the values of $\Delta G_{\text {ads }}^{\circ}$

Table 3. Thermodynamic parameters.

\begin{tabular}{lcc}
\hline Temperature $(\mathrm{K})$ & $K_{\text {ads }}\left(\mathrm{l} \mathrm{mol}^{-1}\right)$ & $\Delta G_{\text {ads }}^{\circ}\left(\mathrm{kJ} \mathrm{mol}^{-1}\right)$ \\
\hline 296 & 333333.3 & -41.21 \\
306 & 125000 & -40.10 \\
316 & 100000 & -40.83 \\
\hline
\end{tabular}

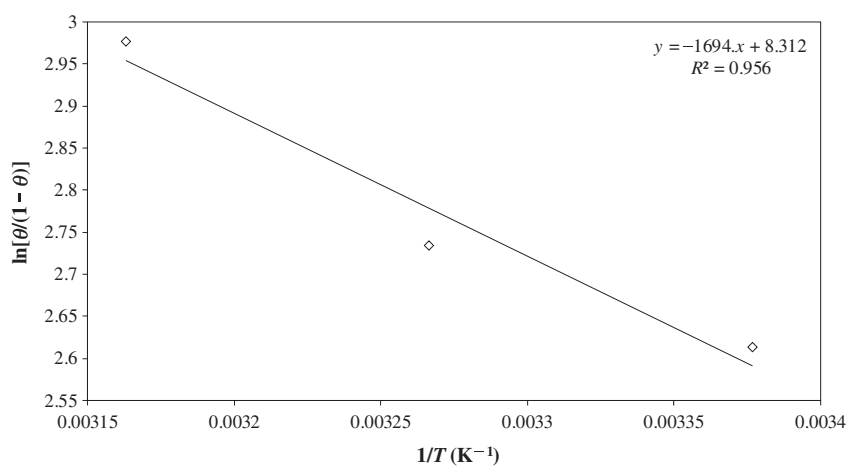

Figure 4. $\ln [\theta /(1-\theta)] v s .1 / T$ for the optimum concentration of FLU.

The entropy of adsorption $\left(\Delta S_{\text {ads }}^{\circ}\right)$ can be calculated using the following equation: ${ }^{31}$

$$
\Delta G_{\mathrm{ads}}^{\circ}=\Delta H_{\mathrm{ads}}^{\circ}-T \Delta S_{\mathrm{ads}}^{\circ} .
$$

By using equation 11 for all temperatures, average $\Delta S_{\text {ads }}^{\circ}$ was found to be $179 \mathrm{~J} \mathrm{~mol}^{-1} \mathrm{~K}^{-1}$. The positive sign of the entropy indicates that an increase in disordering takes places in going from reactants to the metal adsorbed species reaction complex. ${ }^{33}$

\subsection{The effect of temperature}

It is well known that the effect of temperature on the inhibited acid-metal reaction is highly complex, because many changes occur on the metal surface such as rapid etching and desorption of inhibitor and the inhibitor itself may undergo decomposition or rearrangement. ${ }^{34}$

The effect of temperature on the corrosion rate of steel in $1 \mathrm{M} \mathrm{HCl}$ solution in the absence and presence of 200 p.p.m. of FLU was studied at different temperatures (296-316 K) by weight loss measurements. The data of table 2 show that at low concentration IE\% decreases as the temperature rises, but at higher concentrations the trend is opposite.

In acidic solution, the natural logarithm of the corrosion rate $\left(v_{\text {corr }}\right)$ is a linear function with $1 / T$ (Arrhenius equation): ${ }^{35}$

$$
\ln \left(v_{\text {corr }}\right)=\ln A-E_{\mathrm{a}}^{*} / R T,
$$

where $v_{\text {corr }}$ is the corrosion rate determined from the weight loss measurement, $E_{\mathrm{a}}^{*}$ is the apparent activation energy, $A$ is the Arrhenius constant, $R$ is the molar gas constant and $T$ the absolute temperature. The apparent activation energy was determined at optimum concentration of FLU (200 p.p.m.) from the slopes of $\ln v_{\text {corr }} v s$. 1/T graph, depicted in figure 5 . The obtained values of $E_{\mathrm{a}}^{*}$ are listed in table 4.

The obtained $E_{\mathrm{a}}^{*}$ for FLU shows that in the presence of inhibitor the apparent activation energy reduced. This reduction indicates that the adsorption of FLU on the surface of the steel occurred via chemisorption mechanism. ${ }^{36}$ 


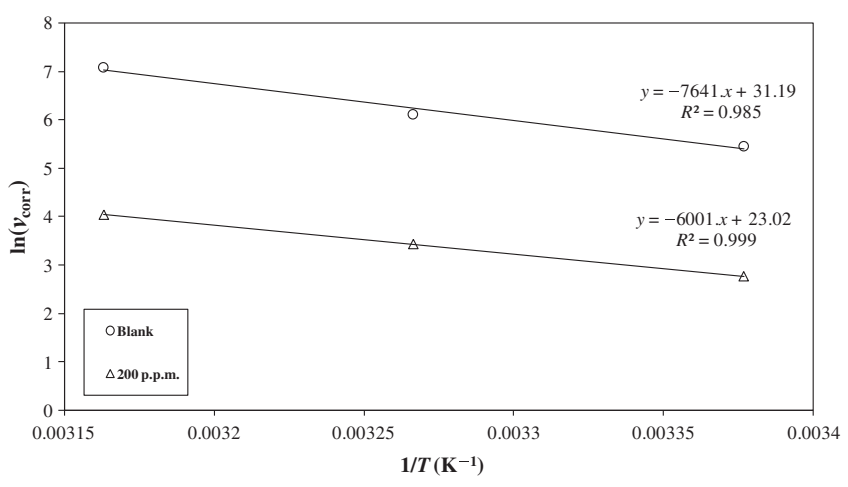

Figure 5. $\ln \left(v_{\text {corr }}\right) v s .1 / T$ for the optimum concentration of FLU.

Table 4. Values of apparent activation energy for steel in $1 \mathrm{M} \mathrm{HCl}$ in the absence and presence of 200 p.p.m. of FLU.

\begin{tabular}{lc}
\hline Concentration (p.p.m.) & $E_{\mathrm{a}}^{*}\left(\mathrm{~kJ} \mathrm{~mol}^{-1}\right)$ \\
\hline 0 & 63.52 \\
200 & 50.35 \\
\hline
\end{tabular}

Table 5. Values of OCP for steel in $1 \mathrm{M} \mathrm{HCl}$ solution in the absence and presence of different concentration of FLU after 30 min immersion.

\begin{tabular}{lc}
\hline Concentration (p.p.m.) & $E_{\text {corr }}(\mathrm{mv})$ \\
\hline 0 & -461 \\
10 & -443 \\
25 & -451 \\
50 & -447 \\
100 & -456 \\
200 & -458 \\
\hline
\end{tabular}

\subsection{Open circuit potential studies}

Open circuit potentials (OCPs) for steel in blank acid and with different concentrations of FLU were measured after 30 min immersion and are shown in table 5.

The classification of a compound as an anodic or cathodic inhibitor is feasible when the OCP displacement is at least $85 \mathrm{mV}$ in relation to the one measured for the blank solution. ${ }^{37,38}$ In our study, the maximum displacement was $18 \mathrm{mV}$ for 10 p.p.m. of FLU. This indicates that FLU is a mixed type inhibitor. It means that the inhibitor reduced the corrosion rate without causing considerable change in the corrosion mechanism. It also shows that the addition of this inhibitor to the solution reduced the anodic dissolution and retarded the cathodic hydrogen evolution reaction. ${ }^{39}$

\subsection{Corrosion inhibition mechanism}

The adsorption process of inhibitor is influenced by the nature of the metal surface, the chemical structure of the organic inhibitor, the distribution of charge in the molecule, the type of aggressive electrolyte and the type of interaction between organic molecules and the metallic surface. ${ }^{40}$ From the structure of the FLU, it is apparent that it can exist in the form of either neutral or cationic molecules in the acidic medium. In the form of cationic molecules, the adsorption could be related to the electrostatic interaction between the protonated compounds and steel surface, negatively charged by the specific adsorption of $\mathrm{Cl}^{-}$ions, and in the form of neutral molecules corrosion inhibition can be attributed to chemisorption. Notably, it is generally accepted that physisorption is preceding stage of chemisorption of inhibitor on metal surface. In other words, chemisorption is always accompanied by physisorption. ${ }^{41,42}$ Therefore, the adsorption of this compound involves two modes of interaction, but mainly chemisorption. This is confirmed by the values of free energy of adsorption, which were around $-40 \mathrm{~kJ} \mathrm{~mol}^{-1}$.

The possible mechanism for chemisorption can be attributed to the donation of $\pi$-electrons by aromatic systems and nonbonding electron pairs of the heteroatoms $(\mathrm{N}, \mathrm{O})$ to form a bond with the vacant d-orbitals of the iron atom on the metal surface, which act as a Lewis acid, leading to the formation of a protective chemisorbed layer.

In hydrochloric acid solution the inhibitor molecules exist as protonated species, which can physically adsorb on the steel surface. The physical adsorption of FLU in hydrochloric acid solution can be explained in the following way:

The charge of the metal surface $(\Phi)$ can be determined by the following equation: ${ }^{43}$

$$
\Phi=E_{\text {corr }}-E_{\mathrm{q}=0},
$$

where $E_{\mathrm{q}=0}$ is the potential of zero charge (PZC). Values of $E_{\text {corr }}$ for steel in $1 \mathrm{M} \mathrm{HCl}$ solution in the absence and presence of different concentration of FLU are shown in table 5.

Values obtained for $E_{\text {corr }}$ in $1 \mathrm{M} \mathrm{HCl}$ solution ranged from -443 to $-461 \mathrm{mV}$ vs. $\mathrm{Ag} / \mathrm{AgCl}$. The PZC of steel in $1 \mathrm{M}$ $\mathrm{HCl}$ solution is $-560 \mathrm{mV}$ vs. SCE $(-516 \mathrm{mV}$ vs. saturated $\mathrm{Ag} / \mathrm{AgCl}),{ }^{44}$ therefore, $\Phi$ has positive value and the steel surface becomes positively charged. On the other hand, the value of $\mathrm{pH}_{\mathrm{iep}}$, which is defined as the $\mathrm{pH}$ of isoelectric point, is around 4 for steel, ${ }^{45}$ so steel surface is positively charged at $\mathrm{pH} 0$, which is the $\mathrm{pH}$ value of $1 \mathrm{M} \mathrm{HCl}$.

It is assumed that in hydrochloric solution, chloride ions first adsorb on the steel/solution interface, through electrostatic attraction forces between these anions and the excess of positive charges at the steel surface. This effect leads to a change on the solution side of the interface, which becomes negatively charged. Thus, protonated FLU molecules are able to adsorb electrostatically on the steel surface previously covered with adsorbed chloride ions. At this point of view, the adsorption of the cationic forms of FLU molecules would be limited by the concentration of the $\mathrm{Cl}^{-}$ions on the metal surface. ${ }^{46}$ 


\subsection{Atomic force microscopy}

AFM is a powerful tool to investigate the surface morphology and it is very useful to determine the film formation on metal surface in corrosion inhibition studies. The average roughness of polished steel before and after immersion in $1 \mathrm{M} \mathrm{HCl}$ solution without the presence of corrosion inhibitor was calculated as 46 and $352 \mathrm{~nm}$, respectively (figure 6a and b). In the presence of 200 p.p.m. of FLU, the average roughness of steel was reduced to $119 \mathrm{~nm}$ (figure 6c).

The lower surface roughness value of the sample immersed in $\mathrm{HCl}$ solution containing FLU compared with the one immersed in $\mathrm{HCl}$ solution in the absence of FLU, confirmed the film formation of the inhibitor over the steel surface, which resulted in reduced corrosion rate and surface degradation of steel surface.

\subsection{Quantum chemical studies}

Quantum chemical calculations have proved to be a very powerful tool for studying corrosion inhibition mechanism. Recently, there has been increasing use of DFT method as a theoretical tool for better understanding the mechanism of corrosion inhibition of organic compounds by several researchers. ${ }^{47,48}$ Thus, a theoretical study was undertaken using DFT method in order to investigate the relation between the inhibitor quantum chemical parameters and its inhibition mechanism.

FLU has a great tendency to get protonated in acidic medium due to the presence of oxygen and nitrogen atoms in its structure. This is can be confirmed by the calculations that show the stability of protonated inhibitor. In order to detect the most favourable active centre for being protonated, optimization of possible structures with different protonated active centres was carried out and it was found that the most probable active centre with lowest energy upon protonation is N6 atom.

The optimized geometry of neutral and protonated form of FLU molecule is shown in figure 7. Bond lengths and bond angles were calculated from optimized geometry and are listed in tables 6 and 7.

Comparing bond length values in neutral and protonated forms showed that protonation did not change the bond lengths drastically. The most changes upon protonation were observed for N5-N4, N4-C10, C10-N6 and C11-N5 bonds. The bond length differences for these bonds were +0.007 , $-0.018,+0.007$ and $-0.013 \AA$, respectively. It is obvious that $\mathrm{C} 10-\mathrm{N} 6$ bond became slightly longer after protonation, which means that the ability of this group as an active centre of adsorption got improved upon protonation. ${ }^{49}$ Comparing bond angle values in neutral and protonated forms showed that highest bond angle changes were attributed to C11-N6-C10, N5-C11-N6, N6-C10-N4, C2-C3-C7, N4-N5-C11, C10-N4-C13 and C7-C13-N4. Bond angle differences for these bonds were $+5.47^{\circ},-4.87^{\circ},-4.54^{\circ}$, $+2.31^{\circ},+2.05^{\circ},-1.16^{\circ}$ and $-1.05^{\circ}$, respectively. The results showed that protonation of N6 did not have significant
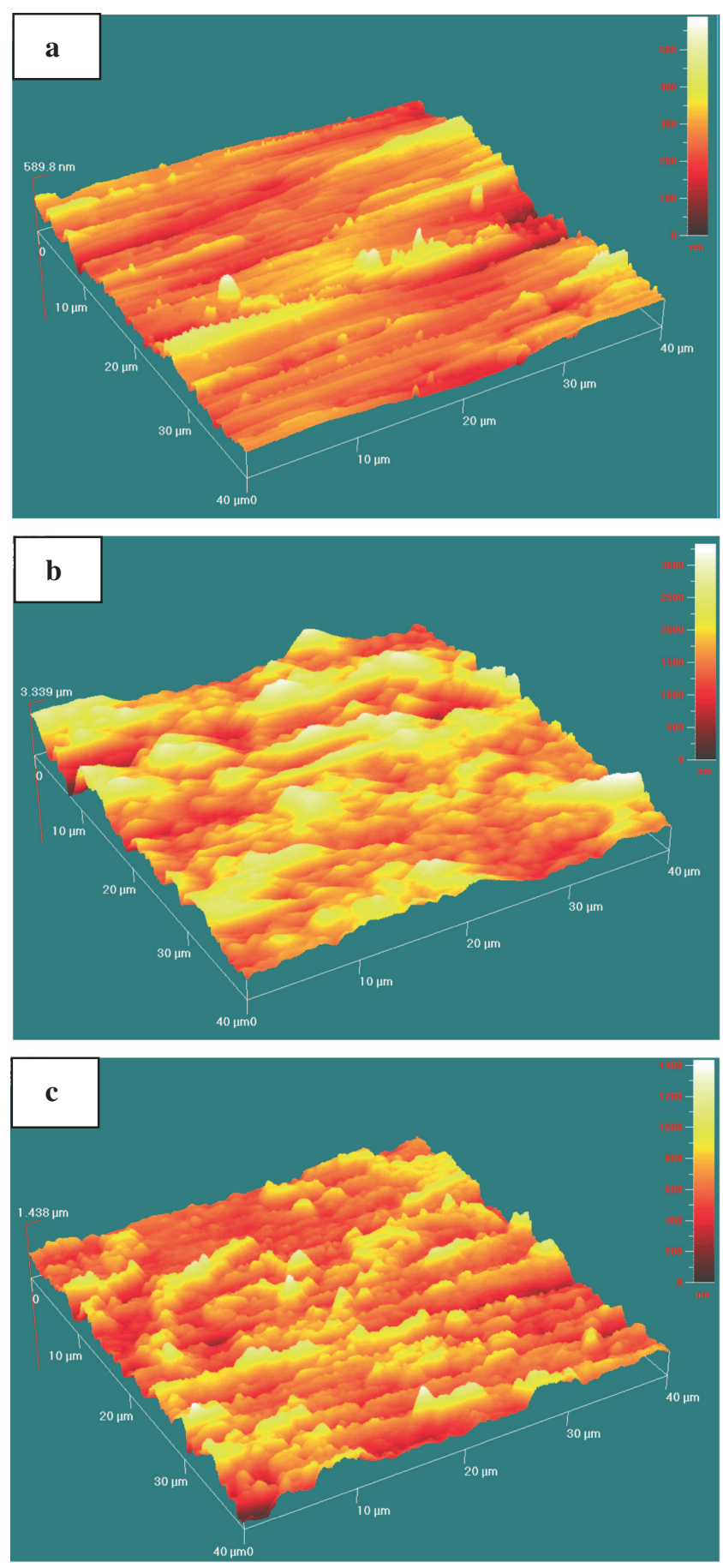

Figure 6. AFM images of the steel surface: (a) before immersion, (b) after $24 \mathrm{~h}$ immersion at $296 \mathrm{~K}$ in $1 \mathrm{M} \mathrm{HCl}$ and (c) after $24 \mathrm{~h}$ immersion at $296 \mathrm{~K}$ in $1 \mathrm{M} \mathrm{HCl}+200$ p.p.m. FLU.

effect on bond angles of FLU molecule and it just affected the bond angles of the triazole ring that contain the N6 atom.

The quantum chemical parameters for neutral and protonated forms of FLU molecule were calculated and are listed in table 8.

According to the frontier molecular orbital (FMO) theory, the chemical reactivity is the function of interaction between 

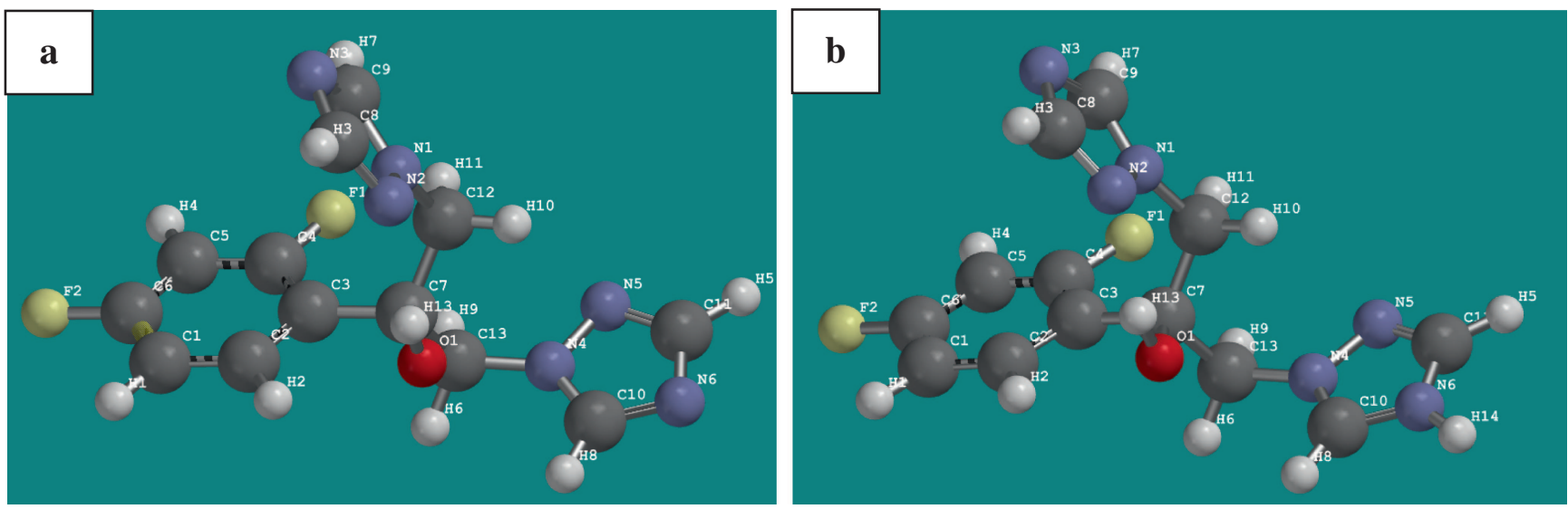

Figure 7. The optimized geometry of (a) neutral and (b) protonated forms of FLU molecule.

Table 6. Bond lengths for neutral and protonated forms of FLU molecule.

\begin{tabular}{lcc}
\hline Bond length $(\AA)$ & Neutral form & Protonated form \\
\hline C1-C2 & 1.394 & 1.394 \\
C2-C3 & 1.400 & 1.400 \\
C3-C4 & 1.398 & 1.398 \\
C3-C7 & 1.539 & 1.539 \\
C4-C5 & 1.387 & 1.387 \\
C4-F1 & 1.364 & 1.363 \\
C5-C6 & 1.388 & 1.388 \\
C6-C1 & 1.387 & 1.387 \\
C6-F2 & 1.357 & 1.359 \\
C7-O & 1.423 & 1.419 \\
C7-C12 & 1.551 & 1.552 \\
C7-C13 & 1.549 & 1.548 \\
C13-N4 & 1.459 & 1.462 \\
N4-C10 & 1.346 & 1.328 \\
C10-N6 & 1.330 & 1.337 \\
N6-C11 & 1.362 & 1.364 \\
C11-N5 & 1.323 & 1.310 \\
N5-N4 & 1.366 & 1.373 \\
C12-N1 & 1.459 & 1.461 \\
N1-N2 & 1.364 & 1.364 \\
N2-C8 & 1.325 & 1.323 \\
C8-N3 & 1.358 & 1.360 \\
N3-C9 & 1.331 & 1.332 \\
C9-N1 & 1.345 & 1.343 \\
\hline
\end{tabular}

HOMO and LUMO levels of the reacting species. $E_{\mathrm{HOMO}}$ is a quantum chemical parameter, which is often associated with the electron donating ability of the molecule. High value of $E_{\text {HOMO }}$ (less negative value) shows the tendency of the molecule to donate electrons to appropriate acceptor molecule with low-molecular orbital energy. Good corrosion inhibitors are usually those organic compounds which not only offer electrons to unoccupied orbital of the metal, but also accept free electrons from the metal. The lower the LUMO energy, the easier the acceptance of electrons from metal surface, which result in decreasing the LUMO-HOMO energy gap and improving efficiency of inhibitor. ${ }^{50-52}$
Table 7. Bond angles for neutral and protonated forms of FLU molecule.

\begin{tabular}{|c|c|c|}
\hline Bond angles $\left({ }^{\circ}\right)$ & Neutral form & Protonated form \\
\hline $\mathrm{C} 1-\mathrm{C} 2-\mathrm{C} 3$ & 122.09 & 122.03 \\
\hline C2-C3-C4 & 116.31 & 116.44 \\
\hline C3-C4-C5 & 123.76 & 123.68 \\
\hline C3-C4-F1 & 119.37 & 119.31 \\
\hline $\mathrm{F} 1-\mathrm{C} 4-\mathrm{C} 5$ & 116.87 & 117.01 \\
\hline C4-C5-C6 & 117.16 & 117.13 \\
\hline C5-C6-F2 & 118.46 & 118.43 \\
\hline F2-C6-C1 & 119.29 & 119.24 \\
\hline $\mathrm{C} 5-\mathrm{C} 6-\mathrm{C} 1$ & 122.25 & 122.33 \\
\hline $\mathrm{C} 6-\mathrm{C} 1-\mathrm{C} 2$ & 118.42 & 118.39 \\
\hline $\mathrm{C} 2-\mathrm{C} 3-\mathrm{C} 7$ & 120.74 & 123.05 \\
\hline C4-C3-C7 & 122.93 & 123.05 \\
\hline C3-C7-O & 111.19 & 111.26 \\
\hline C3-C7-C13 & 107.80 & 107.48 \\
\hline C3-C7-C12 & 112.12 & 112.30 \\
\hline $\mathrm{O}-\mathrm{C} 7-\mathrm{C} 13$ & 105.67 & 105.27 \\
\hline C7-C13-N4 & 114.01 & 112.96 \\
\hline C13-N4-N5 & 121.51 & 120.89 \\
\hline N4-N5-C11 & 102.03 & 104.08 \\
\hline N5-C11-N6 & 115.79 & 110.92 \\
\hline C11-N6-C10 & 101.89 & 107.36 \\
\hline N6-C10-N4 & 110.81 & 106.27 \\
\hline C10-N4-C13 & 128.79 & 127.63 \\
\hline C13-C7-C12 & 110.35 & 110.58 \\
\hline C7-C12-N1 & 111.96 & 111.90 \\
\hline C12-N1-C9 & 129.75 & 129.95 \\
\hline N1-C9-N3 & 110.80 & 110.71 \\
\hline C9-N3-C8 & 102.18 & 102.17 \\
\hline N3-C8-N2 & 115.34 & 115.32 \\
\hline C8-N2-N1 & 102.43 & 102.45 \\
\hline N2-N1-C12 & 120.86 & 120.56 \\
\hline
\end{tabular}

$\Delta E$ (energy gap) is an important parameter as a function of reactivity of the inhibitor molecule towards the adsorption of inhibitor on the metallic surface. As $\Delta E$ decreases (most especially for the cationic species), the reactivity of the molecule increases and as a result the inhibition efficiency of the molecule increases. A molecule with a low energy 
Table 8. Quantum chemical parameters for neutral and protonated forms of FLU molecule.

\begin{tabular}{lcc}
\hline & \multicolumn{2}{c}{ Calculated value } \\
\cline { 2 - 3 } Quantum parameter & Neutral form & Protonated form \\
\hline$\mu(\mathrm{D})$ & 3.11 & 20.2 \\
$\Delta E\left(E_{\mathrm{LUMO}}(\mathrm{ev})-E_{\mathrm{HOMO}}(\mathrm{ev})\right)$ & 6.36 & 5.95 \\
$\Delta N(\mathrm{e})$ & 0.078 & 0.031 \\
\hline
\end{tabular}

gap is more polarizable and is generally associated with the high chemical activity and low kinetic stability and is termed as a soft molecule. ${ }^{53}$ Quantum chemical parameters listed in table 8 reveal that neutral form of FLU has relatively low energy gap. Comparing protonated and neutral forms of FLU shows that FLU molecule in protonated form has lower energy gap $(\Delta E)$ compared to neutral form, which means that in protonated form the inhibitor has higher reactivity towards the steel surface.

Physical adsorption of the organic inhibitor on the metal surface rises as a result of electrostatic attraction between the organic dipole and the charged surface of the metal; therefore, high dipole moment of the molecules favour adsorption ability. ${ }^{54}$ Comparing dipole moments shows that in protonated form dipole moment has increased significantly, so it can be concluded that in protonated form FLU molecule can physically get adsorbed on steel surface.

According to Pearson, ${ }^{55}$ when two systems including metal $(\mathrm{Fe})$ and inhibitor are brought together, electrons will flow from lower $\chi$ (inhibitor) to higher $\chi(\mathrm{Fe})$ until the chemical potentials become equal. As a first approximation, the fraction of electron transferred, $\Delta N$ is given by the following equation:

$$
\Delta N=\left(\chi_{\mathrm{Fe}}-\chi_{\mathrm{inh}}\right) / 2\left(\eta_{\mathrm{Fe}}+\eta_{\mathrm{inh}}\right),
$$

where $\chi_{\mathrm{Fe}}$ and $\chi_{\text {inh }}$ denote the absolute electronegativity of iron and the inhibitor molecule, respectively. $\eta_{\mathrm{Fe}}$ and $\eta_{\mathrm{inh}}$ denote the absolute hardness of iron and the inhibitor molecule, respectively. These quantities are related to electron affinity $(A)$ and ionization potential $(I)$, which are useful for predicting chemical behaviour. ${ }^{56}$

$$
\begin{aligned}
& \chi=(I+A) / 2 \\
& \eta=(I-A) / 2
\end{aligned}
$$

$I$ and $A$ are related to $E_{\mathrm{HOMO}}$ and $E_{\mathrm{LUMO}}$ by following the equations:

$$
\begin{aligned}
& I=-E_{\text {Homo }} \\
& A=-E_{\text {Lumo }}
\end{aligned}
$$

Values of $\chi_{\text {inh }}$ and $\eta_{\text {inh }}$ were calculated by using the values of $I$ and $A$ obtained from quantum chemical calculation. The theoretical values of $\chi_{\mathrm{Fe}}=7.0 \mathrm{eV}$ and $\eta_{\mathrm{Fe}}=0$ were used according to Pearson's electronegativity scale, assuming that for a metallic bulk $I=A$, because they are softer than neutral metallic atoms. ${ }^{55,57}$
$\Delta N$ values, which are the fraction of electrons transferred from inhibitor to the steel surface, were calculated for both neutral and protonated forms (table 8). Values of $\Delta N$ show that inhibition effect resulted from electron donation. According to Lukovits et al, ${ }^{58}$ if $\Delta N<3.6$, the inhibition efficiency increases with increasing electron donating ability at the metal surface. In this study, FLU was the donor of electrons, and the steel surface was the acceptor. This result supports the assertion that the adsorption of inhibitor on the metal surface could occur by donor-acceptor interactions of the $\pi$-electrons and nonbonding electron pairs of the heteroatoms $(\mathrm{N}, \mathrm{O})$ with the vacant d-orbitals of the iron atom on the metal surface.

The Mulliken charge distribution and the HOMO distribution can be used to predict the possible adsorption centres of the inhibitors. Generally, the more negatively charged heteroatom is, the greater is its ability to donate electrons. The more negatively charged regions with major distribution of the HOMO are the most probable adsorption centres. ${ }^{59,60}$ The Mulliken charges for all atoms of neutral and protonated forms of FLU are listed in table 9 and the density distribution of FMOs (HOMO and LUMO) are shown in figures 8 and 9.

From values listed in table 9, it can be seen that the most favourable sites for the interaction between both neutral and protonated molecules of FLU and the metal surface are the following atoms: $\mathrm{O}, \mathrm{N} 3$ and N6, because these atoms have a larger negative charge, suggesting that these active centres with excess charges can act as a nucleophilic reagent. Generally, heteroatoms with larger negative charge have

Table 9. Mulliken charges for all atoms of neutral and protonated forms of FLU molecule.

\begin{tabular}{lrc}
\hline & \multicolumn{2}{c}{ Mulliken charge } \\
\cline { 2 - 3 } Atom & Neutral form & Protonated form \\
\hline C1 & -0.182 & -0.200 \\
C2 & -0.182 & -0.167 \\
C3 & 0.077 & 0.062 \\
C4 & 0.336 & 0.346 \\
C5 & -0.253 & -0.200 \\
C6 & 0.349 & 0.341 \\
C7 & 0.216 & 0.275 \\
C8 & 0.152 & 0.170 \\
C9 & 0.179 & 0.180 \\
C10 & 0.185 & 0.328 \\
C11 & 0.138 & 0.279 \\
C12 & -0.171 & -0.178 \\
C13 & -0.160 & -0.160 \\
N1 & -0.206 & -0.215 \\
N2 & -0.346 & -0.347 \\
N3 & -0.497 & -0.515 \\
N4 & -0.221 & -0.225 \\
N5 & -0.332 & -0.292 \\
N6 & -0.507 & -0.530 \\
O & -0.671 & -0.673 \\
F1 & -0.308 & -0.305 \\
F2 & -0.322 & -0.328 \\
\hline
\end{tabular}


better electron donor ability. ${ }^{60}$ Analysing the HOMO density distributions for both neutral and protonated forms of FLU (figure 8) show that the highest occupied molecule orbital (HOMO) is mostly distributed around benzene ring, this makes it the preferred zone for donating electron and forming a bond with vacant d-orbitals of the iron through its $\pi$ electrons. Overall, HOMO density distribution and Mulliken charges show that $\mathrm{O}, \mathrm{N} 3, \mathrm{~N} 6$ and benzene ring can act as the adsorption centres of the inhibitors, but as the molecule is not planer there is a little chance for N3 to act as an adsorption centre and participate in this process.

$\mathrm{X}$-ray photoelectron spectroscopy (XPS) is one of the tests that can give some information about composition of the organic film formed on the surface. Reviewing the literature shows that some researches have been done on trizaole derivatives in hydrochloric solution by XPS test. These researches have proved that triazole derivatives can chemically interact with steel surface and form a chemisorbed film. ${ }^{61,62}$ They have also shown that $\mathrm{N}-\mathrm{Fe}$ bond may be formed during such adsorption. ${ }^{63}$ These results may support the assertion that adsorption of FLU occurs through $\pi$-electrons, $\mathrm{O}$ and $\mathrm{N}$ atoms.
The LUMO orbital has the most contribution corresponding to anti-bonding characteristic on the ring moiety, which probably facilitates the back donation from the metal surface to the anti-bonding orbitals of the inhibitor. ${ }^{49}$ As shown in figure 9, for neutral form of FLU the LUMO orbitals are mainly distributed on the benzene group, which means that this group besides donating electron to metal surface also facilitates the back donation from the metal surface to the anti-bonding orbitals of the inhibitor. For protonated form, the LUMO orbitals are mainly distributed on one of the triazole rings, which indicate that this ring facilitates the back donation from the metal surface to the anti-bonding orbital's of the inhibitor.

\section{Conclusion}

- Results obtained from the experimental data showed that FLU can act as an effective inhibitor for the corrosion of steel in $1 \mathrm{M} \mathrm{HCl}$ at different temperatures.

- The adsorption of FLU on steel surface in $\mathrm{HCl}$ solution was mainly due to chemisorption at steel/HCl acid interface and obeyed the Langmuir adsorption.

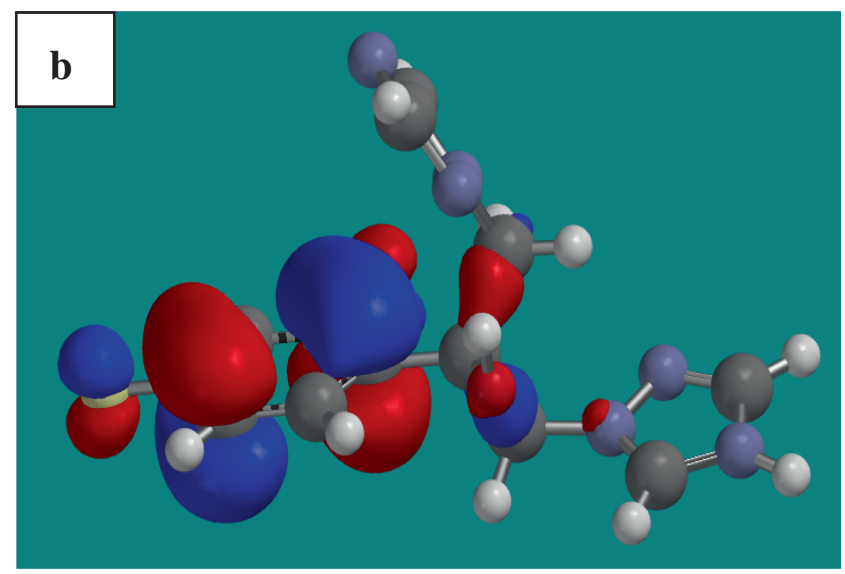

Figure 8. The HOMO density distribution of (a) neutral and (b) protonated forms of FLU molecule.
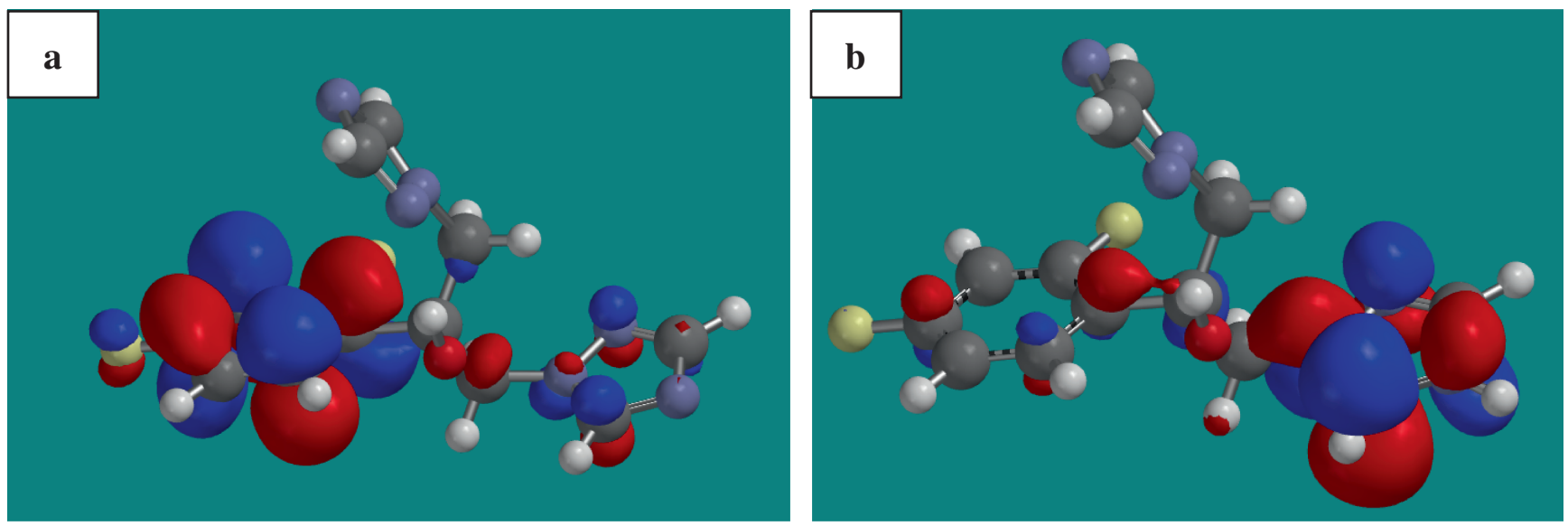

Figure 9. The LUMO density distribution of (a) neutral and (b) protonated forms of FLU molecule. 
- The values of average surface roughness derived from AFM images supported the barrier film formation on steel surface.

- Quantum chemical calculations showed that in adsorption process of FLU molecules, nitrogen and oxygen atoms and benzene ring act as active centres.

- The results obtained in this study indicated that in acidic medium, both neutral and protonated forms of molecule can give better insight into finding the correlation between inhibition effect and molecular structure of an inhibitor.

\section{References}

1. Shukla S K and Quraishi M A 2010 Mater. Chem. Phys. 120 142

2. Li X, Deng S, Fu H and Li T 2009 Electrochim. Acta 544089

3. He X, Jiang Y, Li C, Wang W, Hou B and Wu L 2014 Corros. Sci. 83124

4. Mejeha I M, Nwandu M C, Okeoma K B, Nnanna L A, Chidiebere M A, Eze F C and Oguzie E E, 2011 J. Mater. Sci. 472559

5. Udhayakala P, Rajendiran T V and Gunasekaran S $2012 A d v$. Sci. Res. 371

6. Ghanbari A, Attar M M and Mahdavian M 2010 Mater. Chem. Phys. 1241205

7. Ebenso E E, Arslan T, Kandemirli F, Love I, Ogretir C, Saracoglu M and Umoren S A 2010 J. Quantum Chem. 110 2614

8. Saliyan V R and Adhikari A V 2008 Bull. Mater. Sci. 31 699

9. John S A M, Ali K M and Joseph A 2011 Bull. Mater. Sci. 34 1245

10. Obot I B and Obi-Egbedi N O 2010 Corros. Sci. 52198

11. Gece G 2011 Corros. Sci. 533873

12. Hoseinzadeh A R, Danaee I, Maddahy M H and Rashvand Avei M 2014 Chem. Eng. Comm. 201380

13. Arslan T, Kandemirli F, Ebenso E E, Love I and Alemu H 2009 Corros. Sci. $\mathbf{5 1} 35$

14. Obot I B, Ebenso E E, Obi-Egbedi N O, Afolabi A S and Gasem Z M 2012 Res. Chem. Intermed. 381761

15. Zhang, S, Tao Z, Liao S and Wu F 2010 Corros. Sci. 523126

16. Hassan H H 2007 Electrochim. Acta 531722

17. Obot I B, Obi-Egbedi N O and Umoren S A 2009 Corros. Sci. 511868

18. Obot I B and Obi-Egbedi N O 2008 Colloids Surf. A Physicochem. Eng. Asp. 330207

19. Obi-Egbedi N O, Obot I B and El-Khaiary M I $2011 \mathrm{~J}$. Mol. Struct. 100286

20. Zarrouk A, Zarrok H, Salghi R, Hammouti B, Al-Deyab S S, Touzani R, Bouachrine M, Warad I and Hadda T B 2012 Int. J. Electrochem. Sci. 76353

21. Bilgic S 2007 Appl. Surf. Sci. 2533913

22. Olivares O, Likhanova N V, Gómez B, Navarrete J, LlanosSerrano M E, Arce E and Hallen J M 2006 Appl. Surf. Sci. 252 2894
23. Behpour M, Attaran A M, Ghoreishi S M, Mohammadi N, Hamadanian M, Salavati-Niasari M and Abbasi M 2014 Chem. Eng. Commun. 2011077

24. Zhang J, Gong X L, Yu H H and Du M 2011 Corros. Sci. 53 3324

25. Sastri V S 2011 (New Jersey, John Wiley \& Sons) 111

26. Zhao J and Chen G 2012 Electrochim. Acta 69247

27. Heydari M and Javidi M 2012 Corros. Sci. 61148

28. Jebakumar Immanuel Edison T and Sethuraman M G 2013 ISRN Electrochem. 20131

29. Özdemir O K, Aytac A and Atilla D 2010 J. Mater. Sci. 46 752

30. Obot I B and Obi-Egbedi N O 2010 E.-J. Chem. 7837

31. Hmamou D B, Salghi R, Zarrouk A, Aouad M R, Benali O, Zarrok H, Messali M, Hammouti B, Kabanda M M, Bouachrine M and Ebenso E E 2013 Ind. Eng. Chem. Res. 52 14315

32. Durnie W 1999 J. Electrochem. Soc. 1461751

33. Benali O, Larabi L, Traisnel M, Gengembre L and Harek Y 2007 Appl. Surf. Sci. 2536130

34. Noor E A and Al-Moubaraki A H 2008 Mater. Chem. Phys. 110145

35. Ghasemi O Danaee I, Rashed G R, Rashvand Avei M and Maddahy M H 2013 J. Cent. South Univ. 20301

36. Okafor P C, Liu X and Zheng Y G 2009 Corros. Sci. 51761

37. Sanatkumar B S, Nayak J and Nityananda Shetty A 2012 Int. J. Hydrogen Energy 379431

38. Ferreira E S, Giacomelli C, Giacomelli F C and Spinelli A 2004 Mater. Chem. Phys. 83129

39. Mert B D, Mert M E, Kardas G and Yazici B 2011 Corros. Sci. 534265

40. Lebrini M, Traisnel M, Lagrenée M, Mernari B and Bentiss F 2008 Corros. Sci. 50473

41. Tang Y, Zhang F, Hu S, Cao Z, Wu Z and Jing W 2013 Corros. Sci. 74271

42. Deng S Li X and Fu H 2011 Corros. Sci. 53760

43. Singh A K and Quraishi M A 2010 Corros. Sci. 521529

44. Nofrizal S, Rahim A A, Saad B, Bothi Raja P, Shah A M and Yahya S 2011 Metall. Mater. Trans. A 431382

45. Petermann L B, Doren A, Baroux B and Bellon-Fontaine M N 1995186179

46. Ahamad I and Quraishi M A 2010 Corros. Sci. 52651

47. Bereket G, Öğretir C and Yurt A 2001 J. Mol. Struct. Theochem. 571139

48. Obot I B, Umoren S A and Ebenso E E 2011 Int. J. Electrochem. Sci. 65649

49. Awad M K, Mustafa M R and Abo Elnga M M $2010 \mathrm{~J}$. Mol. Struct. Theochem. 95966

50. Khaled K F 2008 Appl. Surf. Sci. 2551811

51. Fu J, Li S, Wang Y, Cao L and Lu L 2010 J. Mater. Sci. 456255

52. Mistry B M, Patel N S, Sahoo S and Jauhari S 2012 Bull. Mater. Sci. 35459

53. Udhayakala P, Rajendiran T V and Gunasekaran S $2012 \mathrm{~J}$. Comput. Methods Mol. Des. 21

54. Stoyanova A, Petkova G and Peyerimhoff S D 2002 Chem. Phys. 2791 
55. Pearson R G 1988 Inorg. Chem. 27734

56. Pearson R G 1986 Proc. Natl. Acad. Sci. 838440

57. Sastri V S and Perumareddi J R 1997 Corrosion 53617

58. Lukovits I, Kálmán E and Zucchi F 2001 Corrosion 573

59. Marins C, Esteves-Souza A, Martinez-Huitle C A, José C, Rodrigues F, Aparecida M, Maciel M and Echevarria A 2013 Corros. Sci. 67281
60. Gao G and Liang C 2007 Electrochim. Acta 524554

61. Bentiss F, Traisnel M Gengembre L and Lagrenee M 2000 Appl. Surf. Sci. 161194

62. Bentiss F, Traisnel M Gengembre L and Lagrenee M 1999 Appl. Surf. Sci. 152237

63. Bentiss F, Jamab C, Mernari B, Attari H, Kadi L, Lebrini M, Traisnel M and Lagrenée M 2009 Corros. Sci. 511628 